\section{Beyond mortality or being mortal? Challenges in understanding and improving life after ARDS}

\author{
Catherine L Hough
}

In 2009, the National Heart Lung and Blood Institute (NHLBI) of USA convened a workshop to discuss the state of clinical and translational science for acute respiratory distress syndrome (ARDS). The panel reviewed epidemiological studies and clinical trials suggesting marked declines in ARDS-related mortality, and discussed observational studies that described the enduring morbidities of ARDS survivorship. The report of this meeting described the challenges of clinical trials focused on mortality and charged clinical researchers to move 'beyond mortality' (as the report was entitled) by including functional, cost and quality of life outcomes in future research. ${ }^{1}$

Over the last decade, the charge of measuring these long-term outcomes has indeed been realised. Along with observational studies, large interventional trials have begun to include outcome assessment at 6 and 12 months after initial trial enrolment, including four large trials conducted by the NHLBI ARDS Clinical Trials Network. This work, led by Needham and Hopkins, collectively called the ARDSNet Long-Term Outcome Study (ALTOS), has led to the largest cohort of ARDS survivors described to date, confirming the findings of preceding single-centre cohorts ${ }^{2-4}$ by revealing an overwhelming degree of post intensive care unit (ICU) morbidity, with persisting impairments in physical, cognitive and mental health function, and health-related quality of life well below expected. ${ }^{5-7}$

Certainly, ancillary inclusion of longterm outcomes as secondary or exploratory end points provides valuable information. However, proving that specific interventions improve long-term outcomes as well as survival requires primary study end points beyond mortality, such as quality of life or health utilities. While such end points are increasingly common in other fields, there are no large ARDS clinical trials to date with primary outcomes focused on long-term survivorship end points such as quality of life. Since early mortality remains high in ARDS,

Correspondence to Dr Catherine L Hough, Division of Pulmonary and Critical Care Medicine, University of Washington, Seattle, WA 98104, USA; cterrlee@uw.edu
$20 \%$ or more in most randomised trial populations, long-term outcomes would also need to incorporate death in order to reduce false conclusions from competing risks and informative differential missingness. Composite outcomes such as quality-adjusted life years (QALYs) represent a validated approach to this problem. QALYs are calculated from measurement of health utilities over time, using instruments such as the Medical Outcomes Study Short Form, ${ }^{8}$ the Health Utility Index $^{9}$ or the EQ-5D. ${ }^{10}$ While using QALYs as a primary outcome measure is appealing, offering insight into quality of survivorship and potentially increasing power to detect differences between study groups, there are also significant barriers to their incorporation into clinical trials. Notably, while these generic measures such as the EQ-5D have been recommended for use in survivors of critical illness, ${ }^{11}$ they do not assess many domains that may be important in this population, and are likely incomplete assessments of quality of life. ${ }^{12}$ Furthermore, the valuations used to determine health utility (such as the time trade-off approach for EQ-5D) have not been specifically validated in survivors of critical illness, which threaten the calculation of QALYs. ${ }^{13}$ Additional barriers to using QALYs as primary outcomes in critical care clinical trials include increased costs and duration of trials, loss to follow-up, statistical challenges, and difficulties of interpretation if the intervention affects mortality and morbidity in opposing directions. ${ }^{14}$ Another key barrier is that little is known about specific risk factors for decreased health utility after ARDS, which are needed to identify high-risk patient groups and potential targets for intervention.

In Thorax, Brown and colleagues tackle this key knowledge gap. ${ }^{15}$ Using hospital data from ARDS Network clinical trials and survey data from ALTOS, investigators sought to determine the association of a large number of factors with health utility scores measured 6 months after ARDS. This combined data set was especially rich in indicators of severity of illness and ICU treatments measured early in the course of critical illness, and also included patient demographics, comorbidities and a crude assessment of functional status. Health utility was measured by the EQ-5D in a range from -0.11 (state worse than death) to 1 (perfect health). ${ }^{13}$ A total of 645 patients participated in 6-month outcome assessment and formed the primary analytical cohort; nearly 30\% of the 1176 trial participants died before this time. The median EQ-5D health utility score was quite low $(0.77$, IQR $0.51-0.83)$, and no variables directly associated with acute illness (eg, treatments, severity of illness or markers of acute pulmonary pathology) were associated in the primary analysis. Seven factors were independently associated with health utility: category of functional independence (home and independent; home with informal care or requiring professional help), age, sex, ethnicity, current smoking status, body mass index and AIDS. No other comorbidities were significantly associated with health utility score after ARDS. The $\mathrm{R}^{2}$ for this model was low, suggesting that the variables did not explain much of the variability of health utility.

The authors conducted a number of additional analyses. First, they added variables available before hospital discharge to the model, which identified hospital length of stay as an independent predictor of poorer health utility. Next, the cohort was expanded to include all patients who died before 6 months; these patients were given a health utility score of 0 . In this cohort, severity of illness (indicated by APACHE score) was a significant predictor of health utility, along with age and functional status. Finally, restricting analysis to the 195 patients who had retrospective EQ-5D assessments (reporting on prehospital status after critical illness), baseline EQ-5D was the most significant predictor of 6-month health utility. Overall, the authors concluded that health utility among survivors of ARDS was not associated with features of acute illness or its treatments. Instead, health utility was associated with baseline demographics such as age and sex, and 'lifestyle factors' such as smoking and obesity. Additionally, a crude assessment of functional statusrequiring no help, informal help or professional help-was a key predictor of health utility in all models.

The finding that pre-ICU factors are the most important determinants of post-ICU health utility is novel in the ARDS population, and is consistent with emerging reports across various ICU populations. For example, a recent study of over 1000 survivors of a clinical trial of renal replacement therapy demonstrated that most had 
low EQ-5D utility scores 60 days later, and that the only significant predictors of health utility were location of residence prior to admission (a surrogate for functional independence, similar to ALTOS) and hospital length of stay. ${ }^{16}$ Indeed, health before critical illness, whether measured as quality of life, ${ }^{17}$ functional dependence ${ }^{18}$ or comorbid disease, ${ }^{19}$ has been demonstrated as the key predictor of the quality of survivorship after ICU. This is not entirely surprising, as trajectories of pre-ICU functional decline are generally not expected to improve after critical illness. Additionally, the EQ-5D is dominated by measures of function, with domains in mobility, self-care, usual activities, pain/discomfort and anxiety/depression; patients who are unable to perform activities of daily living (ADL) independently would be expected to have low EQ-5D scores at baseline. Overall, the authors' pragmatic approach to capturing functional independence is an important step forward, and further attempts using tools such as ADLs or measures of frailty will likely advance the field even further.

The absence of association between acute illness factors, such as severity of illness and ICU treatments, and health utility may be explained by a number of hypotheses. First, all patients in this cohort had a high severity of illness: all had ARDS, all required mechanical ventilation and all had significant hypoxaemia. It is possible that ARDS-associated factors are linked to poorer outcome, but a cohort limited to patients with ARDS is unable to test such a hypothesis. Second, the association between acute illness factors and health utility may be modified by baseline health; additional stratified or restricted analyses may reveal important associations in subgroups. This approach may be crucial in order to identify the patients most likely to respond to treatments targeted at reducing post-ICU sequelae. Third, acute illness factors may be most important earlier in recovery. Fourth, there could be important effects of critical illness on health utility which are not captured by the EQ-5D. And finally, there may be no important association between acute illness factors and health utility; after all, neither cognitive function nor post-traumatic stress disorder - contributors to health utility-have proven associations with severity of illness. $^{20}$ The lack of association between most comorbidities and health utilities in the present study can be similarly explained, with particular attention to the fact that many severe comorbidities were excluded from the original ARDS Network studies (such as chronic lung disease and severe cirrhosis) and that patients with comorbid diseases were much more likely to die before 6-month assessment.

Identifying predictors of health utility after ARDS represents important progress, and the authors' proposal to use these predictors in developing tailored interventions, particularly for patients who smoke or who are obese, is certainly a valuable next step forward. However, given how poorly the kinds of variables examined by Brown et al predict health utility across a large cohort of ARDS survivors, perhaps our field first needs to look backwards. We need to understand how to detect the groups for whom critical illness represents a causal change to their long-term health status, so that we can identify etiological pathways and test specific interventionsthis will likely require exploring the heterogeneity of baseline factors, acute illness factors and the contributors to health status itself. We need more precise measurement of baseline health and trajectory at the time of ICU admission, since this is convincingly associated with both mortality and post-ICU morbidity. And we need to advance our patient-centred care, especially for those whose outcomes are unlikely to improve with targeted treatments. After all, as Atul Gawande writes in Being Mortal, 'our interventions, and the risks and sacrifices they entail, are justified only if they serve the larger aims of a person's life'. ${ }^{21}$ With these steps, our field may truly move beyond mortality.

Twitter Follow Catherine Hough @terri_hough

Competing interests None declared.

Provenance and peer review Commissioned; externally peer reviewed.

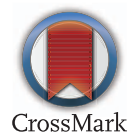

To cite Hough CL. Thorax 2017;72:302-303.

Published Online First 9 February 2017

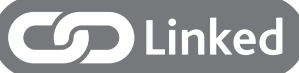

- http://dx.doi.org/10.1136/thoraxjnl-2016-208560

Thorax 2017;72:302-303.

doi:10.1136/thoraxjnl-2016-209082

\section{REFERENCES}

1 Spragg RG, Bernard GR, Checkley W, et al. Beyond mortality: Future clinical research in acute lung injury. Am J Respir Crit Care Med 2010;181:1121-7.

2 McHugh LG, Milberg JA, Whitcomb ME, et al. Recovery of function in survivors of the acute respiratory distress syndrome. Am I Respir Crit Care Med 1994;150:90-4.
3 Herridge MS, Cheung AM, Tansey CM, et al. One-year outcomes in survivors of the acute respiratory distress syndrome. N Engl I Med 2003:348:683-93.

4 Herridge MS, Tansey CM, Matte A, et al. Functional disability 5 years after acute respiratory distress syndrome. N Eng/ J Med 2011;364:1293-304.

5 Needham DM, Dinglas VD, Bienvenu OJ, et al. One year outcomes in patients with acute lung injury randomised to initial trophic or full enteral feeding: Prospective follow-up of eden randomised trial. BMJ 2013:346:f1532.

6 Needham DM, Dinglas VD, Morris PE, et al. Physical and cognitive performance of patients with acute lung injury 1 year after initial trophic versus full enteral feeding. Eden trial follow-up. Am J Respir Crit Care Med 2013;188:567-76.

7 Needham DM, Wozniak AW, Hough CL, et al. Risk factors for physical impairment after acute lung injury in a national, multicenter study. Am J Respir Crit Care Med 2014;189:1214-24.

8 Brazier J, Roberts J, Deverill M. The estimation of a preference-based measure of health from the sf-36. J Health Econ 2002;21:271-92.

9 Furlong WJ, Feeny DH, Torrance GW, et al. The health utilities index (hui) system for assessing health-related quality of life in clinical studies. Ann Med 2001;33:375-84.

10 Walters SJ, Brazier JE. Comparison of the minimally important difference for two health state utility measures: EQ-5D and SF-6D. Qual Life Res 2005;14:1523-32.

11 Needham DM, Dowdy DW, Mendez-Tellez PA, et al. Studying outcomes of intensive care unit survivors: Measuring exposures and outcomes. Intensive Care Med 2005;31:1153-60.

12 Lim WC, Black N, Lamping D, et al. Conceptualizing and measuring health-related quality of life in critical care. J Crit Care 2016;31:183-93.

13 Shaw JW, Johnson JA, Coons SJ. Us valuation of the eq-5d health states: development and testing of the d1 valuation model. Med Care 2005;43:203-20.

14 Ferguson ND, Scales DC, Pinto R, et al. Canadian Critical Care Trials G. Integrating mortality and morbidity outcomes: Using quality-adjusted life years in critical care trials. Am J Respir Crit Care Med 2013;187:256-61.

15 Brown SM, Wilson E, Presson AP, et al. Predictors of 6 -month health utility outcomes in survivors of acute respiratory distress syndrome. Thorax 2017:72: 311-317.

16 Johansen KL, Smith MW, Unruh ML, et al., Network VNARFT. Predictors of health utility among 60-day survivors of acute kidney injury in the veterans affairs/national institutes of health acute renal failure trial network study. Clin I Am Soc Nephrol 2010;5:1366-72.

17 Feemster LC, Cooke CR, Rubenfeld GD, et al. The influence of hospitalization or intensive care unit admission on declines in health-related quality of life. Ann Am Thorac Soc 2013;12:35-45

18 Ferrante LE, Pisani MA, Murphy TE, et al. Factors associated with functional recovery among older intensive care unit survivors. Am I Respir Crit Care Med 2016;194:299-307.

19 Lone NI, Gillies MA, Haddow C, et al. Five-year mortality and hospital costs associated with surviving intensive care. Am J Respir Crit Care Med 2016;194:198-208.

20 Herridge MS, Moss M, Hough CL, et al. Recovery and outcomes after the acute respiratory distress syndrome (ARDS) in patients and their family caregivers. Intensive Care Med 2016:42:725-38.

21 Gawande A. Being mortal: medicine and what matters in the end. 1st edn. Metropolitan Books/ Henry Holt and Company, 2014. 